\title{
Compilação dos inquéritos epidemiológicos em saúde bucal no Brasil de 1986 a 2015: Principais aspectos metodológicos
}

\author{
Compilation of epidemiological surveys in oral health in Brazil from 1986 to 2015: Main \\ methodological aspects
}

Compilación de encuestas epidemiológicas en salud bucal en Brasil de 1986 a 2015: Principales aspectos metodológicos

\author{
Maria Helena Ribeiro De Checchi \\ ORCID: https://orcid.org/0000-0002-8431-6533 \\ Universidade Federal do Amazonas, Brasil \\ E-mail: mariahelenard@ hotmail.com \\ Carla Fabiana Tenani \\ ORCID: https://orcid.org/0000-0001-7203-2763 \\ Universidade Estadual de Campinas, Brasil \\ E-mail: carlatenani@ hotmail.com \\ Fernanda Dandara Marques Gomes de Morais \\ ORCID: https://orcid.org/0000-0001-7356-4577 \\ Universidade Estadual de Campinas, Brasil \\ E-mail: fernandad.marques@hotmail.com \\ Marcelo de Castro Meneghim \\ ORCID: https://orcid.org/0000-0003-2673-3627 \\ Universidade Estadual de Campinas, Brasi \\ E-mail: meneghim@fop.unicamp.br \\ Antonio Carlos Pereira \\ ORCID: https://orcid.org/0000-0003-1703-8171 \\ Universidade Estadual de Campinas, Brasil \\ E-mail: apereira111@gmail.com
}

\begin{abstract}
Resumo
Introdução: os inquéritos epidemiológicos em saúde bucal servem como subsídio de políticas públicas e, até o momento, não encontramos publicações sobre uma compilação de todos os importantes inquéritos realizados no Brasil. Objetivo: compilar todas as metodologias empregadas nos levantamentos epidemiológicos em saúde bucal realizados no Brasil entre os anos de 1986 a 2015. Métodos: compilação realizada a partir de buscas em livros, periódicos, artigos nas bases de dados LILACS, MEDLINE, BBO e SCIELO, como também relatórios finais dos estudos. Os levantamentos epidemiológicos em saúde bucal realizados no Estado de São Paulo foram avaliados nos anos 1998, 2002 e 2015, além dos efetuados nos anos 1986, 1993, 1996, 2003 e 2010 com abrangência nacional. Resultados: compiladas as metodologias empregadas foram se modificando e ampliando o escopo dos problemas estudados. Há evidências de melhora na elaboração de processos de trabalho dos levantamentos epidemiológicos, principalmente no que se refere à abrangência da população pesquisada, calibração e aferição de resultados. Conclusão: os avanços nos critérios metodológicos de investigação podem ser atribuídos à força de políticas públicas em saúde bucal implementadas no Brasil nas últimas décadas. Além disso, verifica-se a constante participação de instituições acadêmicas, num crescente processo de interação do conhecimento científico com a prática. Palavras-chave: Epidemiologia; Saúde bucal; Inquéritos epidemiológicos; Aplicações da epidemiologia.
\end{abstract}

\begin{abstract}
Introduction: oral health epidemiological surveys use public policies as a subsidy, until the time when publications on a compilation of all surveys conducted in Brazil are not published. Objective: to compile all the methodologies used in the main epidemiological surveys on oral health carried out in Brazil between the years 1986 to 2015. Methods: compilation based on researches in books, periodicals, articles in LILACS, MEDLINE, BBO and SCIELO databases, as well as final reports of studies. Evaluations over the surveys carried out in the State of São Paulo in 1998, 2002 and 2015 as well as those carried out in the years 1986, 1993, 1996, 2003 and 2010 covering the entire country. Results: compiled the methodologies used were modifying themselves and expanding the scope of the problems studied. There is evidence of improvement in the elaboration of work procedures in epidemiological surveys, mainly in what refers to the coverage of the researched population, calibration and measurement of results. Conclusion: the advances in the
\end{abstract}


methodological criteria on researches can be attributed to the strength of public policies regarding oral health that have been implemented in Brazil over the last decades. In addition, there is a constant participation of academic institutions in a growing process of interaction between scientific knowledge and practice.

Keywords: Epidemiology; Oral health; Epidemiological surveys; Epidemiologic measurements.

\section{Resumen}

Introducción: las encuestas epidemiológicas en salud bucal sirven de subsidio a las políticas públicas y, hasta el momento, no hemos encontrado publicaciones sobre una recopilación de todas las encuestas importantes realizadas en Brasil. Objetivo: recopilar todas las metodologías utilizadas en las encuestas epidemiológicas sobre salud bucal realizadas en Brasil entre 1986 y 2015. Métodos: recopilación realizada a partir de búsquedas en libros, revistas, artículos en las bases de datos LILACS, MEDLINE, BBO y SCIELO, así como informes finales de los estudios. Se evaluaron las encuestas epidemiológicas de salud bucal realizadas en el Estado de São Paulo en 1998, 2002 y 2015, además de las realizadas en 1986, 1993, 1996, 2003 y 2010 con cobertura nacional. Resultados: recopilados, las metodologías utilizadas fueron cambiando y ampliando el alcance de los problemas estudiados. Existe evidencia de mejora en la elaboración de procesos de trabajo en encuestas epidemiológicas, especialmente en lo que se refiere al alcance de la población encuestada, calibración y medición de resultados. Conclusión: los avances en los criterios metodológicos de investigación se pueden atribuir a la fortaleza de las políticas públicas en salud bucal implementadas en Brasil en las últimas décadas. Además, existe la participación constante de las instituciones académicas, en un proceso creciente de interacción entre el conocimiento científico y la práctica.

Palabras clave: Epidemiología; Salud bucal; Encuestas epidemiológicas; Aplicaciones de la epidemiología.

\section{Introdução}

Levantamentos ou inquéritos epidemiológicos são estudos transversais, podendo ser conceituados como instrumentos de gestão utilizando metodologia específica. Têm finalidade de conhecer a extensão e severidade de problemas em saúde, diagnosticar as necessidades acumuladas, auxiliar na determinação de atividades prioritárias a serem implementadas, além de avaliar programas (Pereira e Silva, 2009). Os dados coletados em um levantamento epidemiológico podem ser utilizados para comparações momento futuro (Pereira, 2000).

Os inquéritos odontológicos abrangem condição de saúde oral, necessidade de tratamento em comunidades, gera dados básicos confiáveis para desenvolvimento de programas em saúde bucal nacionais, estaduais e municipais, propiciando racionalizar recursos humanos, materiais e financeiros (Pereira e Silva, 2009). Levantamentos epidemiológicos bucais aferem condições orais de comunidades inferindo sobre suas necessidades de assistência, a partir da produção de dados fidedignos obtidos através de metodologia específica. São apoio para a construção de políticas públicas onde investimentos financeiros e recursos humanos obedeçam à lógica de ordenamento equânime (Pinto, 2000). Esses levantamentos fornecem uma gama de informações apuradas que podem propiciar condições para controlar as mudanças dos níveis ou padrões da doença (WHO, 1997).

Enquanto internacionalmente, países nórdicos e Inglaterra possuíam bases com dados de prevalência da cárie dentária desde as primeiras décadas deste século, o primeiro dado nacional no Brasil, obtido de dados secundários, foi publicado em 1980 e apresentava uma média do índice de dentes cariados perdidos e obturados (CPOD) aos 12 anos de idade de 7,3 dentes. Entretanto, tal informação traduzia compilação de várias pesquisas isoladas, não podendo ser considerada pesquisa com dados primários (Pinto, 1983; Oliveira et al., 1998; Narvai et al., 1999).

Inquéritos brasileiros em saúde bucal de grande abrangência, obtidos através de estudos primários, foram realizados de 1986 a 2015, e evidenciaram o declínio da cárie dentária, o que pode ser atribuído, em parte aos seguintes aspectos: diferenças metodológicas, intervenções governamentais em saúde bucal implementadas no Brasil nas últimas décadas, mudança no critério de diagnóstico, o aumento da oferta de dentifrícios fluoretados à população, consolidação do SUS, reorganização da prática odontológica com ênfase em promoção e prevenção, introdução de procedimentos coletivos e processo de municipalização com incentivo a participação popular, o que corrobora com a ideia da saúde bucal como indissociável da saúde geral do indivíduo (Rosendo et al., 2017). 
Considerando que o conhecimento e diagnóstico sobre a saúde bucal da população brasileira tenham se desenvolvido de maneira discreta ao longo dos anos (Martins et al., 2005), a análise dos 8 levantamentos epidemiológicos em saúde bucal ocorridos no Brasil nas últimas décadas (de 1986 a 2015) mostra uma evolução quanto a características metodológicas como: o aumento da abrangência da população examinada em zonas urbanas em 1993 e, a partir de 2003, a inclusão da zona rural; aumento do escopo de doenças bucais examinadas; caracterização socioeconômica a partir do estudo de 2003, voltando a atenção para a influência dos determinantes sociais da população avaliada; o levantamento de 2010 (Brasil, 2010) considerado o mais abrangente, introduziu questões subjetivas, ratificando importância na abrangência de escopo para o planejamento dos gestores de saúde.

Assim, devido à escassez de estudos científicos sobre o tema e sua importância estratégica para definição de políticas públicas em saúde, o presente estudo teve como propósito compilar todas as metodologias empregadas nos levantamentos epidemiológicos em saúde bucal realizados no Brasil entre os anos de 1986 a 2015. Ao se elencar as metodologias de forma sistematizadas, acredita-se que seja possível apoiar o estabelecimento aprimorado de novos inquéritos.

\section{Métodos}

A aprovação em Comitê de Ética em Pesquisa não foi necessária para a elaboração deste artigo pois se trata de revisão da literatura.

Este estudo foi realizado por meio de buscas ativas sob a temática, em livros, periódicos e artigos nacionais na base de dados nacionais e de língua portuguesa: MEDLINE, LILACS, BBO e SciELO, bem como em documentos e relatórios finais de levantamentos (Brasil, 1986; Brasil, 1996; Pinto, 1996; Brasil, 2004; Brasil, 2012; Pereira et al., 2016), todos de base nacional ou regional (estado de SP) de 8 inquéritos realizados desde 1986, até o último em 2015.Trata-se de estudo quali- quantitativo.

Foram considerados os inquéritos epidemiológicos em saúde bucal realizados no Estado de São Paulo em 1998, 2002 e 2015, acrescidos dos inquéritos nacionais realizados nos anos de 1986, 1993, 1996, 2003, 2010.

A busca pelos artigos foi realizada de agosto a dezembro de 2017, sob os seguintes termos nas línguas portuguesa e inglesa: levantamento epidemiológico OR inquérito AND saúde bucal AND metodologia AND Brasil OR Brazil AND Oral Health AND Epidemiological surveys AND metodology, em artigos publicados no período de 1968 a 2017. Dos 164 artigos encontrados, foram selecionados 8 artigos, 1 dissertação de mestrado e 1 tese de doutorado. Não foi encontrado nenhum artigo que tratasse especificamente sobre as metodologias referentes a todos os inquéritos em saúde bucal realizados no país e citados no presente estudo.

Para delimitar o objeto de estudo e o campo de investigação desse estudo, como critérios de inclusão, optou-se por selecionar apenas produções na forma de artigos publicados em periódicos nacionais brasileiros, tendo sido considerado o ano de 1986 como o ano base. Esta opção se fez por ter ocorrido, nesse ano, os primeiros registros formais e científicos a respeito de estudos pioneiros de inquéritos em saúde bucal no Brasil.

A Figura 1 mostra que, para o processo de seleção para estudos elegíveis, a pesquisa da literatura inicialmente atingiu 174 artigos sendo que, ao final, 8 artigos foram elegidos. Não foi encontrado nenhum artigo que tratasse especificamente sobre todas as metodologias referentes a todos os inquéritos em saúde bucal incluídos neste estudo.

A análise dos estudos encontrados foi feita de forma descritiva e realizada em duas etapas. A primeira incluiu: tipo de estudo, ano e variáveis estudadas (amostra, população-alvo, faixas etárias, variáveis socioeconômicas/demográficas, e de saúde bucal - CPOD, ceo-d, DAI, AG, PIP, CPI, calibração, locais de exames, diagnóstico de cárie). A segunda etapa compreendeu a análise das principais diferenças dentre as metodologias utilizadas nos levantamentos. 
Figura 1. Fluxograma da seleção dos estudos incluídos.

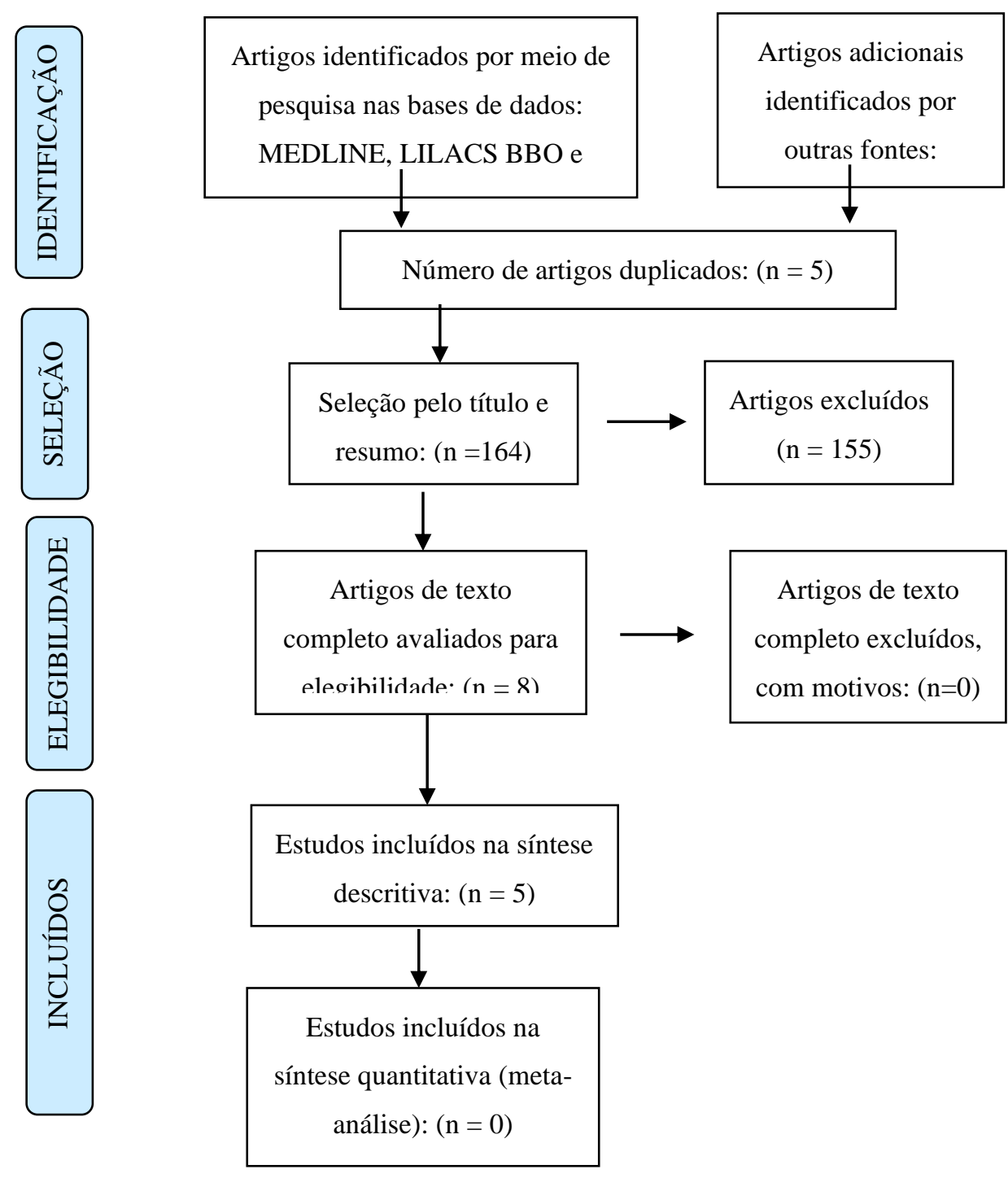

Fonte: Autores.

A partir desta ferramenta de organização de estudo, foi possível observar que apesar do substancial número de manuscritos encontrados ao início da pesquisa, poucos se enquadraram no escopo do estudo. Esta metodologia de estruturação proporciona clareza no ordenamento de cada etapa.

\section{Resultados}

No ano de 1986 foram coletados dados referentes à cárie dentaria, doença periodontal e necessidade de prótese. Em 1993 e 1996, as informações foram referentes somente à cárie. Nos anos de 2003, 2010 e 2015 foram acrescidas informações sobre auto percepção e acesso ao serviço.

A tabela 1 retrata os principais aspectos metodológicos dos inquéritos em saúde bucal realizados no estado de São Paulo nos anos de 1998, 2002 e 2015. Em relação à população paulista, destaca-se o aumento quanto a proporção da amostra, bem como em relação à abrangência de localidades ao longo dos levantamentos verificados. No inquérito realizado em 2015 , não foi averiguada a faixa etária infantil, mantendo-se a prevalência de adolescentes, adultos jovens e idosos nos outros levantamentos 
verificados. As variáveis socioeconômicas começam as ser consideradas apenas no inquérito do ano de 2015 . Todos os levantamentos (1998, 2002 e 2015) contaram com calibração preconizada pela OMS. No levantamento de 2002, o CPI foi realizado apenas sobre os códigos 0,1 e 2 .

Tabela 1. Aspectos metodológicos dos levantamentos epidemiológicos em saúde bucal realizados no Estado de São Paulo nos anos de 1998, 2002 e 2015.

\begin{tabular}{|c|c|c|c|c|c|c|c|}
\hline LSB/ano & $\begin{array}{l}\text { População } \\
\text { Amostra }\end{array}$ & $\begin{array}{l}\text { Pessoas } \\
\mathbf{N}^{\circ}\end{array}$ & $\begin{array}{c}\text { Faixas } \\
\text { Etárias/Id } \\
\text { ades } \\
\text { Índices }\end{array}$ & Variáveis & Calibração & $\begin{array}{c}\text { Local } \\
\text { (exame) }\end{array}$ & $\begin{array}{c}\text { Diagnóstico de Cárie } \\
\text { (critério) }\end{array}$ \\
\hline 1998 & $\begin{array}{l}133 \text { municípios } \\
\text { do Estado de } \\
\text { São Paulo de } \\
\text { pequeno, } \\
\text { grande e médio } \\
\text { porte }\end{array}$ & $\begin{array}{c}\mathrm{N}=96 \\
\text { element } \\
\text { os } \\
\text { amostrai } \\
\text { s por } \\
\text { extrato }\end{array}$ & $\begin{array}{c}5 \text { a } 12 \\
18 \\
35-4465- \\
74\end{array}$ & $\begin{array}{c}\text { - Cárie dentária, } \\
\text { doença } \\
\text { periodontal, má } \\
\text { oclusão, } \\
\text { fluorose } \\
\text { dentária, uso de } \\
\text { prótese dentária } \\
\text { e necessidades } \\
\text { de tratamento } \\
\text { odontológico }\end{array}$ & $\begin{array}{c}\text { examinadores } \\
\text { nas } 24 \text { regiões } \\
\text { do Estado } \\
(\text { OMS }) / \text { discor } \\
\text { dância de } 10 \text { a } \\
15 \% .\end{array}$ & $\begin{array}{l}\text { Iluminação } \\
\text { natural. } \\
\text { Ambiente } \\
\text { escolar }\end{array}$ & $\begin{array}{l}\text { Cavidade evidente, } \\
\text { tecido amolecido, } \\
\text { descoloração do } \\
\text { esmalte ou de parede, } \\
\text { restauração temporária } \\
\text { (exceto ionômero de } \\
\text { vidro para dentes } \\
\text { decíduos) }\end{array}$ \\
\hline 2015 & $\begin{array}{c}\text { Conglomerado } \\
\text { s e } \\
\text { Macrorregiões } \\
\text { (6) em todo } \\
\text { Estado de SP } \\
1^{\text {a }} \text { fase: } \\
\text { sorteados } 178 \\
\text { municípios, } \\
\text { mais a Capital } \\
\text { do Estado } \\
\text {-2 } \text { fase sorteio: } \\
390 \text { setores (2 } \\
\text { para } 177 \\
\text { municípios e } \\
36 \text { para SP }\end{array}$ & $\begin{array}{c}\mathrm{N}=1756 \\
0\end{array}$ & $\begin{array}{c}15 \text { a } 19 \\
35 \text { a } 44 \\
65 \text { anos ou } \\
\text { mais }\end{array}$ & $\begin{array}{l}\text { Socioeconômico } \\
\text {, utilização de } \\
\text { serviço, } \\
\text { autopercepção, } \\
\text { e capital social }\end{array}$ & $\begin{array}{c}\text { Modelo } \\
\text { preconizado } \\
\text { pela OMS } \\
\text { (WHO, 1997) }\end{array}$ & $\begin{array}{l}\text { Domiciliar } \\
\text { (sob luz } \\
\text { natural) }\end{array}$ & $\begin{array}{c}\text { Índice preconizado } \\
\text { pela OMS (WHO, } \\
1997)\end{array}$ \\
\hline
\end{tabular}

Fontes: Relatório final do Levantamento Epidemiológico em Saúde Bucal: Estado de São Paulo, (1998). São Paulo - (1999); Relatório Final SB São Paulo Condições de Saúde Bucal no Estado de São Paulo (2002); Relatório Final SB São Paulo Pesquisa Estadual de Saúde Bucal (2015). Autores.

Já a Tabela 2 apresenta os principais aspectos metodológicos dos levantamentos epidemiológicos, com abrangência nacional, ocorridos de 1986 a 2015. Em relação à amostra, somente a partir do ano de 2003, foi incluída população rural. Até o levantamento de 1996, só eram incluídos escolares como número de pessoas para os inquéritos. Entretanto, até 1996, não há registro de idosos participantes dos levantamentos. Somente a partir do inquérito realizado em 2003, foi incluída a variável 
socioeconômica. Até o levantamento de 1996, não foram registrados os níveis de concordância entre os calibradores participantes. Realizaram-se exames clínicos (sob luz natural) em ambientes não clínicos, com exceção de 1986, onde os exames aconteceram também em alguns consultórios odontológicos, tal informação não consta no levantamento de 1996 (Roncalli, 1998; Brasil, 2004; Brasil, 2010; Frias, 2015).

Já a Tabela 2 apresenta os principais aspectos metodológicos dos levantamentos epidemiológicos, com abrangência nacional, ocorridos de 1986 a 2015. Em relação à amostra, somente a partir do ano de 2003, foi incluída população rural. Até o levantamento de 1996, só eram incluídos escolares como número de pessoas para os inquéritos. Entretanto, até 1996, não há registro de idosos participantes dos levantamentos. Somente a partir do inquérito realizado em 2003, foi incluída a variável socioeconômica. Até o levantamento de 1996, não foram registrados os níveis de concordância entre os calibradores participantes. Realizaram-se exames clínicos (sob luz natural) em ambientes não clínicos, com exceção de 1986, onde os exames aconteceram também em alguns consultórios odontológicos, tal informação não consta no levantamento de 1996 (Roncalli, 1998; Brasil, 2004; Brasil, 2010; Frias, 2015). 
Tabela 2. Aspectos metodológicos dos inquéritos em saúde bucal realizados em âmbito nacional nos anos de 1986, 1993, 1996, 2003 e 2010.

\begin{tabular}{|c|c|c|c|c|c|c|c|}
\hline $\begin{array}{l}\text { LSB/ } \\
\text { ano }\end{array}$ & $\begin{array}{c}\text { População } \\
\text { Amostra }\end{array}$ & $\begin{array}{c}\text { Pessoas } \\
\mathbf{N}^{\circ}\end{array}$ & $\begin{array}{c}\text { Faixas } \\
\text { Etárias/Idades } \\
\text { Índices } \\
\end{array}$ & Variáveis & Calibração & $\begin{array}{l}\text { Local/ } \\
\text { exame }\end{array}$ & $\begin{array}{c}\text { Diagnóstico } \\
\text { de Cárie/ } \\
\text { critério } \\
\end{array}$ \\
\hline 1986 & $\begin{array}{c}\text {-Zona urbana } \\
\text { (16 capitais) } \\
\text { onde havia } \\
\text { FSESP* } \\
120 \text { estudantes } \\
\text { por escola } 16 \\
\text { domicílios } \\
\text { (setor } \\
\text { censitário) } \\
\mathrm{N}=23180 \\
\end{array}$ & $\begin{array}{c}\mathrm{N}=\text { sem } \\
\text { informação }\end{array}$ & $\begin{array}{c}6,7,8,9,10,11,12 \\
15 \text { a } 19 \\
35 \text { a } 44 \\
50 \text { a } 59\end{array}$ & $\begin{array}{l}\text { Renda, sexo, } \\
\text { CPOD e ceo-d, } \\
\text { CPI, prótese } \\
\text { total, } \\
\text { atendimento, } \\
\text { hábitos de } \\
\text { higiene bucal } \\
\text { (não divulgados) }\end{array}$ & $\begin{array}{l}\text { Supervisores e } \\
\text { futuros } \\
\text { multiplicadores }\end{array}$ & $\begin{array}{c}\text { Ambiente } \\
\text { escolar sob } \\
\text { luz natural e } \\
\text { alguns } \\
\text { consultórios } \\
\text { odontológicos }\end{array}$ & $\begin{array}{l}\text { Retenção de } \\
\text { Sonda } \\
\text { Exploradora }\end{array}$ \\
\hline 1993 & $\begin{array}{c}\text {-Zona urbana } \\
\text {-estudantes de } \\
\text { escolas do } \\
\text { SESI } \\
\text {-escolas } \\
\text { públicas } \\
\text { N=110640 }\end{array}$ & $\begin{array}{c}\mathrm{N}=110604 \\
\text { (escolares) } \\
\mathrm{N}=58450 \\
(\mathrm{SESI}) \\
\mathrm{N}=52190 \\
\text { (escolas } \\
\text { públicas) }\end{array}$ & $\begin{array}{c}\text { Idades índices } \\
3-14\end{array}$ & CPOD e ceo & $\begin{array}{c}\text { Sem } \\
\text { evidências }\end{array}$ & $\begin{array}{c}\text { Pátio de } \\
\text { escolas (sob } \\
\text { luz natural) }\end{array}$ & $\begin{array}{l}\text { Retenção de } \\
\text { Sonda } \\
\text { Exploradora }\end{array}$ \\
\hline 1996 & $\begin{array}{c}\text {-Zona urbana } \\
\text { /Amostragem } \\
\text { (OMS e MS } \\
\text { Brasil) } \\
\mathrm{N}=30240\end{array}$ & $\begin{array}{c}\mathrm{N}=30240 \\
\text { (escolares) }\end{array}$ & $\begin{array}{c}\text { Idades índices } \\
6,7,8,9 \\
10,11,12\end{array}$ & $\begin{array}{c}\text { Sexo; } \\
\text { CPOD e ceo }\end{array}$ & $\begin{array}{c}\text { Calibração: } \\
\text { nacional, } \\
\text { regional, } \\
\text { estadual, e } \\
\text { municipal. } \\
\text { Sem } \\
\text { concordância e } \\
\text { resultados }\end{array}$ & $\begin{array}{l}\text { Não consta } \\
\text { informação } \\
\text { no relatório }\end{array}$ & $\begin{array}{c}\text { Exame } \\
\text { visual } \\
\text { (dente } \\
\text { cariado com } \\
\text { cavidade } \\
\text { em nível de } \\
\text { dentina) }\end{array}$ \\
\hline 2003 & $\begin{array}{l}\text {-Urbana e rural } \\
\text {-macrorregiões } \\
\text { e porte } \\
\text { municipal. } \\
\mathrm{N}=175000\end{array}$ & $\mathrm{~N}=108921$ & $\begin{array}{c}5 \text { e } 12 \\
0-18 \text { meses } \\
15 \text { a } 19 \\
35 \text { a } 44 \\
65 \text { a } 74\end{array}$ & $\begin{array}{l}\text { Socioeconômico; } \\
\text { acesso; } \\
\text { autopercepção; } \\
\text { sexo; etnia; flúor } \\
\text { água; } \\
\text { CPOD/ceo/CPI/ } \\
\text { PIP/AG/Dean/ } \\
\text { DAÍ/ má } \\
\text { oclusão/próteses/ } \\
\text { alteração } \\
\text { tecido/mole }\end{array}$ & $\begin{array}{l}\text { Calibração: } \\
\text { nacional, } \\
\text { regional, } \\
\text { estadual, e } \\
\text { municipal. } \\
\text { Cálculo } \\
\text { (KAPPA). } \\
\text { Resultados não } \\
\text { foram } \\
\text { publicados }\end{array}$ & $\begin{array}{l}\text { Ambiente não } \\
\text { clínico (sob } \\
\text { luz natural) }\end{array}$ & $\begin{array}{c}\text { Exame } \\
\text { visual } \\
\text { (dente } \\
\text { cariado com } \\
\text { cavidade } \\
\text { evidente) }\end{array}$ \\
\hline 2010 & $\begin{array}{l}\text { Conglomerados } \\
\text { (26 capitais) } \\
\text { Distrito Federal } \\
\text { e ( } 150 \text { /interior) } \\
\mathrm{N}=37519\end{array}$ & $\mathrm{~N}=37519$ & $\begin{array}{c}5 \text { e } 12 \\
15 \text { a } 19 \\
34 \text { a } 45 \\
65 \text { a } 74\end{array}$ & $\begin{array}{l}\text { Socioeconômico, } \\
\text { utilização de } \\
\text { serviços } \\
\text { odontológicos, } \\
\text { autopercepção }\end{array}$ & $\begin{array}{c}\text { Modelo } \\
\text { preconizado } \\
\text { pela OMS } \\
\text { (WHO, 1993), } \\
\text { e índice } \\
\text { KAPPA }\end{array}$ & $\begin{array}{l}\text { Domiciliar } \\
\text { (sob luz } \\
\text { natural) }\end{array}$ & $\begin{array}{c}\text { Índice } \\
\text { preconizado } \\
\text { pela OMS } \\
\text { (WHO, } \\
1997)\end{array}$ \\
\hline
\end{tabular}

Fontes: Brasil, Ministério da Saúde, 1988. Brasil, Ministério da Saúde, (1996). Pinto VG (1993/1996); SESI, 1993. Roncalli AG, (1998). Martins et al., (2005). Brasil, Ministério da Saúde, 2004. Relatório Final Levantamento Epidemiológico em Saúde Bucal SBBrasil, (2010). Relatório Final Levantamento Epidemiológico em Saúde Bucal no Estado de São Paulo SBSP, (2015). * FSESP Fundação de Serviços de Saúde Pública. Autores.

Quanto à calibração, no ano de 1986 foi realizada por supervisores e futuros multiplicadores. Nos anos de 1996 e 2003 ocorreu em todo território nacional, sendo que apenas em 2003 houve o cálculo de concordância e dos resultados, porém não publicados. Em 2010 e 2015 foram utilizados os modelos preconizados pela OMS (WHO, 1997). 
A retenção da sonda exploradora foi o critério de diagnóstico de cárie utilizado nos levantamentos realizados nos anos de 1986 e 1993. Posteriormente, em 1996 e 2003 foram realizados exames visuais, considerando dente cariado os com cavidade evidente, e por fim nos últimos levantamentos, 2010 e 2015, foram utilizados os índices preconizados pela OMS (WHO, 1997).

\section{Discussão}

Levantamentos epidemiológicos em SB no Estado de São Paulo.

\section{Levantamento - 1998}

No final da década de 90, por não haver registro de apontamentos recentes, a Secretaria da Saúde do Estado São Paulo (SES/SP) entendeu contundente inteirar-se sobre as condições de saúde bucal experimentadas pela população do Estado.

Neste sentido, propôs a realização de levantamento para investigar as condições de saúde bucal da população estratificada nas idades índices de 5 a 12,18, 35 a 44 anos de idade vinculados às unidades das redes de ensino pública e privada do Estado e idosos de 65 a 74 anos de idade.

Foram exploradas as seguintes variáveis: cárie dentária, doença periodontal, oclusão dentária, fluorose dentária, uso de prótese dentária, bem como as necessidades de tratamento odontológico e de prótese dentária.

Os exames foram realizados em escolas em locais com luz natural abundante, por conglomerado, uma adaptação da ficha simplificada da OMS foi utilizada como instrumento de coleta. Todos os examinadores foram calibrados e as atividades foram planejadas e executadas de forma descentralizada. Para criação e análise do banco de dados utilizou-se o programa EPIBUCO.

A partir desse inquérito foi possível descrever o cenário estadual em saúde bucal. A partir de variáveis constantes no inquérito verificou-se distância considerável entre a realidade e as metas propostas pela OMS para o ano 2000. No entanto, o escopo de informações nele contidas pôde subsidiar ações de setores públicos e privados com vistas a prosperar o quadro.

\section{Levantamento - 2002}

Buscou-se através desta pesquisa de abrangência estadual a complementação das informações logradas no Levantamento Epidemiológico em Saúde Bucal - Estado de São Paulo - 1998 (Brasil, 2002a).

Além de variáveis sócio econômicas, e auto percepção, esta pesquisa abordou de forma segmentada em idades índices (18 a 36 meses, 5, 12, 15 a 19, 35 a 44 e 65 a 74 anos) as seguintes patologias: cárie, doença periodontal fluorose, má oclusão bem como a necessidade de uso de prótese dentária (Brasil, 2002a).

A população de 5 anos de idade foi alvo de pesquisa de prevalência de alterações gengivais, enquanto que a prevalência de alteração periodontais foi na de 12 a 65-74 anos. Já a prevalência de oclusopatias teve como público de i nvestigação indivíduos de 5 a 15-19 anos, enquanto que a fluorose dentária foi pesquisada em indivíduos de 12 a 15 19 anos. A estimativa de necessidade de uso de prótese dentária foi abordada nas faixas etárias de 15 a 65-74 anos. As unidades amostrais primárias (municípios) foram definidas a partir de estratificação de acordo com número de habitantes (Brasil, 2002a).

Para o quesito presença de cárie dentária, nas idades índices de 5 e de 12 anos, calculou-se o tamanho da amostra segundo suposição de ataque de cárie estabelecida no levantamento de 1996 do MS. Já no que tange os grupos etários de 15 a 19, 35 a 44 e 65 a 74 anos, foi previsto o tamanho da amostra a partir das estimativas de cárie produzidas em 1986, também do MS (Brasil, 2002a). 
Para a idade índice de 12 anos o inquérito ocorreu em unidades de ensino, sorteados de forma ponderada. Definiu-se o número de 20 estabelecimentos por municípios. Os elementos amostrais foram identificados pela técnica de amostragem casual sistemática. No caso dos municípios com prevalência de 50 mil habitantes, definiu-se como setores censitários as (UAS) e os estabelecimentos da rede pública e privada de ensino para as crianças de 5 e 12 anos de idade.

Para este inquérito unidades amostrais primárias (UAP) foram selecionadas nos municípios. Já para representatividade de unidades amostrais secundárias (UAS) foram definidas quadras para áreas urbanas, e vilas para áreas rurais.

O modelo de ficha empregado no SB200 foi aproveitado e utilizado como instrumento neste levantamento.

Foram selecionados 5 examinadores por município. A calibração foi desenvolvida em 24 horas de atividades para obtenção de concordâncias intra examinadores e inter examinadores.

\section{Levantamento - 2015}

Em meio ao cenário epidemiológico de emergência em saúde pública (Machado et al., 2017), causado pela epidemia de Zika vírus, ocorreu o levantamento epidemiológico de base domiciliar SB SP2015. Teve como objetivo conhecer as condições de saúde bucal nas populações de 15-19, de 35-44 e de 65 anos e mais (Frias, 2015) no estado de São Paulo no ano de 2015. As idades de 5 e 12 anos de idade foram investigadas em outro estudo complementar desenvolvido em 2014 (Fase I do Levantamento Epidemiológico Saúde Bucal Nos Municípios - Base Escolar de 5 e 12 anos) e seguiram as normas do SB Brasil 2010 (Brasil, 2010).

O SBSP2015 foi uma realização da Secretaria de Estado da Saúde (SES-SP) e contou com financiamento da Fundação de Amparo à Pesquisa do Estado de São Paulo (FAPESP). A Coordenação Técnico-Científica da pesquisa incorporou docentes da FOP/Unicamp, FOUSP e da Secretaria Estadual de Saúde de SP (Pereira et al., 2016).

Mais de 550 profissionais participaram efetivamente do estudo, sendo todos os examinadores participantes calibrados (Pereira et al., 2016). A amostra complexa foi composta por conglomerados e macrorregiões do estado, em 163 municípios.

As seguintes variáveis foram abordadas: cárie, doença periodontal, uso e necessidade de prótese, maloclusão, além da condição socioeconômica, utilização de serviços odontológicos, autopercepção de saúde bucal e capital social e acesso. Utilizou-se as recomendações da OMS (WHO, 1997), com pequenas alterações propostas pela FSP-USP. Os exames clínicos bucais foram realizados nos domicílios sob luz natural e calibrados (Pereira et al., 2016).

Levantamentos epidemiológicos em SB no Brasil de 1986, 1993, 1996, 2003 e 2010

\section{Levantamento - 1986}

Em 1986, quando o cenário político-sanitário constituía-se ainda do pós queda do regime de ditadura militar, à medida que a sociedade voltava a atuar no sistema político, a saúde pública conquistava a reforma sanitarista, culminando na criação do SUS (Martins et al., 2005), ocorreu o primeiro levantamento epidemiológico em saúde bucal de âmbito nacional.

$\mathrm{Na}$ oportunidade foram levantados dados referentes à cárie dental, doença periodontal e necessidades de prótese sob critérios metodológicos da Organização Mundial de Saúde (OMS) (Martins et al., 2005).

Previamente a esta data, ocorreram estudos brasileiros locais como os realizados por Grego (Grego, 1968) a respeito de anormalidades oclusais, raça e higiene bucal no município de Araraquara/SP, Souza sobre atendimento dentários em escolares de 8 a 12 anos, e de Colli e colaboradores, (Colli et al., 1975) sobre a saúde de um grupo de adolescentes na cidade de São Paulo, porém não possuindo caráter de inquérito.

No intuito de obter informações sobre as condições de saúde bucal da população brasileira ocorreu pioneiramente o primeiro levantamento epidemiológico em saúde bucal na história da saúde pública brasileira em nível ministerial (Zanetti, 
1993). Este foi vinculado ao Programa de Prevenção de Cárie (PRECAD) (já extinto) sob um cenário político de redemocratização do país (Nova República), apresentando um crescimento e amadurecimento do Movimento Sanitário, ocorrência da VIII Conferência Nacional de Saúde e da criação da Divisão Nacional de Saúde Bucal Brasileira (DNSB) (Roncalli, 2012).

Neste cenário foi divulgado e publicado o primeiro relatório sobre o levantamento epidemiológico de abrangência nacional brasileira pela DNSB do MS. Entretanto, este levantamento foi realizado somente em capitais (16 representativas das 27 existentes) (Brasil, 1986). Todavia, este pode ser considerado como a primeira estimativa mais confiável da realidade epidemiológica brasileira em saúde bucal Brasil, 2002).

Os exames odontológicos (com uso de sonda exploratória) ocorreram em alguns consultórios privados e em ambiente escolar (Brasil, 1986). Os problemas investigados foram à cárie, doença periodontal - Índice Comunitário de Necessidade de Tratamento Periodontal (CPITN), uso e necessidade de prótese, além de ter se obtido informações sobre utilização de serviços odontológicos e hábitos de higiene bucal, porém, nenhum resultado foi apresentado no relatório sobre este último item (São Paulo, 1999).

As amostras foram selecionadas abrangendo 120 estudantes de escolas de $1^{\circ}$ e $2^{\circ}$ graus, sendo a faixa etária compreendendo crianças de 6 a 12 anos e os demais exames em domicílios e consultórios odontológicos para as faixas de adultos e idosos (15-19, 35-44 e 50-59 anos), respectivamente.

Interessante apontar que tal levantamento foi voltado para avaliações de condições de saúde bucal sob uma perspectiva exclusivamente profissional, ignorando como tais percepções ocorriam na população (Buischi, 2000).

\section{Levantamento de 1993}

Logo após um conturbado momento político no Brasil, onde no ano de 1992, o então presidente da república Fernando Collor de Melo sofreu processo de impeachment e em meio à implementação do plano Real para controle da inflação no país, um levantamento ocorreu no ano de 1993 e realizado pelo Serviço Social da Indústria (SESI). Este levantamento fez parte do programa preventivo de doenças bucais, em crianças de escolas em cidades interioranas, porém não havendo abrangência de todos os Estados brasileiros13. Este foi financiado por um convênio com o Departamento Nacional (DN) do SESI, além de financiamento externo da Kolynos do Brasil para impressão de seus relatórios (Pinto, 1993).

Neste levantamento avaliou-se somente a doença cárie com auxílio de sonda exploratória.

\section{Levantamento - 1996}

Em tempo de instituição das Normas Operacionais Básicas (NOB's), que o outorgaram aos municípios a responsabilidade por atender às necessidades do cidadão na Atenção Básica, ocorre o $2^{\circ}$ Levantamento de base nacional financiado pelo Ministério da Saúde.

Foi realizado após 10 anos da primeira pesquisa nacional (1986), através da coordenação de saúde bucal (COSAB) do MS em conjunto com a Associação Brasileira de Odontologia (ABO) nacional, Conselho Federal de Odontologia (CFO) e Secretarias Estaduais de Saúde. Teve como objetivo verificar a prevalência de cárie no país, apesar disto sofreu diversas críticas juntamente com reclamações de Faculdades não integradas ao processo (Roncalli, 1998).

Este inquérito foi executado no primeiro mandato de Fernando Henrique Cardoso, com a democracia representativa aparentemente consolidada, e a saúde bucal como Área Técnica no Ministério da Saúde (Roncalli, 2012).

Neste levantamento sob a perspectiva profissional, houve plano amostral realizado somente em capitais. Sendo o mais abrangente em diagnóstico e com a realização de calibração prévia de examinadores, foi avaliada somente a doença cárie com auxílio de sonda exploratória, em ambientes não clínicos. 


\section{Levantamento - 2003}

Apresentando pouca mudança na política macroeconômica, há a criação da Política Nacional de Saúde Bucal (Brasil Sorridente), priorizada no conjunto de políticas públicas (Brasil, 2012). Nesse cenário foi realizado o SB Brasil 2003 (Brasil, 2004).

Este inquérito ocorreu sob preceitos da Organização Mundial da Saúde20. Incluiu aspectos subjetivos como características socioeconômicas, autopercepção em saúde bucal, acesso e satisfação a serviços odontológicos. Houve realização de exames bucais em amostra com cálculo estratificado por macrorregiões e por número de habitantes, em regiões urbanas e incluindo rurais de todos os estados brasileiros. Contou, além disso, com parceria de Faculdades de Odontologia (São Paulo, 1998; Brasil, 2004).

O SB Brasil 2003 - Condições de Saúde Bucal da População Brasileira -apresentou um maior critério amostral com um maior rigor metodológico em relação aos anteriores. Pesquisou alterações de tecidos moles não analisadas até então. Ainda foram coletadas índices referentes à perda de inserção periodontal (IPC), alterações gengivais (AG), má oclusão sob o índice de estética dental (DAÍ) e fluorose pelo índice de Dean (OMS, 1997). Foi realizada prévia calibração dos examinadores, incluindo descrição de cálculo Kappa (Brasil, 2004).

Contou ainda com dados sobre uso e necessidade de próteses totais e removíveis e pontes fixas.

Este inquérito em termos gerais, não obstante o fato de expandir os problemas e melhorar substancialmente a metodologia empregada, ainda assim sofreu algumas críticas pela comunidade acadêmica, uma vez que apresentou falhas no banco de dados em etapas fundamentais como registro e arquivamento dos documentos.

Levantamento - 2010

No ano de 2010 foi desenvolvida a Pesquisa Nacional de Saúde Bucal - SB Brasil 2010, um estudo transversal multicêntrico com abordagem qualitativa e de abrangência nacional sob coordenação do MS. Para que isso ocorresse, secretarias estaduais e municipais de saúde das cinco macrorregiões brasileiras participaram com o apoio de entidades de classe odontológicas, institutos de pesquisa e universidades.

Este inquérito foi executado com proposta metodológica sugerida como estratégia para o desenvolvimento do eixo de vigilância em saúde da Política Nacional de Saúde Bucal18. Importante salientar que tal levantamento foi inserido na Política Nacional de Saúde Bucal (PNSB) (Roncalli, 2012).

Executado em 26 capitais estaduais, no Distrito Federal e em cento e cinquenta municípios do interior de diferentes portes populacionais, contou com o trabalho realizado por volta de dois mil profissionais do Sistema Único de Saúde (SUS) das três esferas governamentais.

Quanto aos principais aspectos metodológicos, houve uma evolução significativa em comparação aos levantamentos anteriores especificamente relacionada à pesquisa realizada somente em domicílios de áreas urbanas, como modificações quanto a avaliação da necessidade de prótese, índice CPI e acréscimo de índices como traumatismo dental. Sua amostra abordou capitais que foram consideradas como domínios, onde foram incluídos 150 municípios do interior, sob uma divisão de 30 por região.

Em termos gerais, houve uma evolução das metodologias aplicadas nos diferentes inquéritos brasileiros de 1986 a 2015. Além disso, comparações dos resultados dos levantamentos sob nítidas diferenças metodológicas, como amostragem, faz surgir a necessidade de maior rigor metodológico, que propicie maior fidedignidade dos dados

Nesse sentido, avaliar as séries históricas dos levantamentos epidemiológicos ocorridos no Brasil, suas dificuldades e avanços alcançados ocorridos até presente momento, torna-se fundamental para que haja uma análise e comparação servindo de subsídio para o planejamento das ações de saúde bucal nacionais. 
Tais levantamentos apresentaram avanços nos critérios metodológicos de investigação dos principais problemas em saúde bucal no Brasil. As metodologias nessas últimas três décadas ampliaram seu escopo e aumentaram o rigor. Tais avanços podem ser atribuídos à força de políticas públicas em saúde bucal implementadas no país nas últimas décadas. Além disso, verifica-se a constante participação de instituições acadêmicas, num crescente processo de interação do conhecimento científico com a prática.

O avanço metodológico dos levantamentos epidemiológicos em saúde bucal não desconsidera ou invalida a grande contribuição trazida pelos primeiros inquéritos aplicados. O estabelecimento de novas estruturas e ordenamentos nesta modalidade de pesquisa foi, ao longo dos anos, sendo aprimorada espelhando-se em pontos assertivos e reformulando-se em aspectos imprecisos.

A utilização de inquéritos epidemiológicos, prática empreendida tanto no Brasil quanto em diversos outros países, representa estratégia relevante como forma de vigilância da saúde bucal. Mesmo a luz do potencial uso do e-SUS, a utilização dessa ferramenta de pesquisa tem um ganho adicional que diz respeito a capacidade de aferir dados de indivíduos não usuários do SUS.

Os levantamentos devem ser reprodutíveis a qualquer tempo para que possam ser subsidiar melhoria efetiva da gestão dos recursos disponíveis. Neste sentido, os principais levantamentos epidemiológicos em território nacional contribuíram para o aprimoramento das políticas públicas em saúde bucal implementadas no país nas últimas décadas.

Apesar da relevância sanitária, dados de levantamentos epidemiológicos com diferenças metodológicas não podem ser comparáveis ao longo do tempo e nem entre as comunidades. Entretanto, há ainda grande potência inexplorada nos inquéritos no que se refere a trazer para a análise questões de saúde bucal relevantes ainda não investigadas.

\section{Conclusão}

Conclui-se que, ao longo das últimas décadas, os levantamentos epidemiológicos de base nacional foram escassos e frequentemente utilizaram metodologias diferentes, tanto os realizados em São Paulo, quanto a nível nacional. A despeito disto, apresentaram avanços nos critérios metodológicos, bem como no escopo de investigação dos principais problemas em saúde bucal no Brasil.

Nesse sentido, o presente estudo oportuniza, por meio da apresentação de sua compilação, que futuros inquéritos possam ampliar ainda mais tanto seus desenhos metodológicos quanto seus quesitos de abordagem e exploração.

Sendo assim, para trabalhos futuros sugere-se que exista um aprimoramento do rigor metodológico, ao mesmo tempo em que sejam introduzidas novas perspectivas, novos olhares abarcando diferentes paradigmas estruturantes da saúde bucal em todo território nacional.

\section{Referências}

Brasil, Ministério da Saúde, Secretaria de Políticas de Saúde, Área Técnica de Saúde Bucal (1996). Levantamento epidemiológico de cárie dentária: banco de dados. www.saude.gov.br.

Brasil. Ministério da Saúde - CONASS 20 anos (2002). A força dos estados na garantia do direito a saúde. http://bvsms.saude.gov.br/bvs/publicacoes/progestores/20anos1.pdf.

Brasil. Ministério da Saúde - Coordenação Nacional de Saúde Bucal (2010). Projeto SB Brasil 2010: projeto técnico. http://www.sbbrasil2010.org.

Brasil. Ministério da Saúde - Coordenação Nacional de Saúde Bucal (2004). Projeto SB Brasil 2003: condições de Saúde Bucal da população Brasileira. http://bvsms.saude.gov.br/bvs/publicacoes/projeto_sb2004.pdf.

Brasil. Ministério da Saúde - Coordenação Nacional de Saúde Bucal (2010). Projeto SB Brasil 2010: projeto técnico. http://www.sbbrasil2010.org.

Brasil. Ministério da Saúde - Divisão Nacional de Saúde Bucal (1988). Levantamento Epidemiológico em Saúde Bucal: Brasil, zona urbana. 1986. Série C: Estudos e Projetos,137p. http://www.saude/saudebucal. 
Research, Society and Development, v. 10, n. 8, e53410817614, 2021

(CC BY 4.0) | ISSN 2525-3409 | DOI: http://dx.doi.org/10.33448/rsd-v10i8.17614

Brasil. Ministério da Saúde (2012). SB Brasil 2010: pesquisa nacional de saúde bucal: resultados principais. Brasília. http://dab.saude.gov.br/CNSB/sbbrasil/arquivos/projeto_sb2010_relatorio_final.pdf.

Brasil. Secretaria de Estado da Saúde de São Paulo e FSP-USP (2002a). Núcleo de Estudos e Pesquisas de Sistemas de Saúde - NEPESS/FSP. http://www.saude.sp.gov.br/resources/ses/perfil/profissional-da-saude/grupo-tecnico-de-acoes-estrategicas-gtae/saude-bucal/artigos-e-teses/estudosepidemiologicos/estudosepidemiologicos/condicoes_de_saude_bucal_-_2002.pdf.

Brasil. Secretaria de Políticas de Saúde, Ministério da Saúde (2001). Projeto SB2000. Área Técnica de Saúde Bucal, Departamento de Atenção Básica, Condições de saúde bucal da população brasileira no ano 2000: manual do coordenador. Brasília: Ministério da Saúde.http://bvsms.saude.gov.br/bvs/publicacoes/condSB_man_exam.pdf

Buischi, Y. P. (2000). Promoção de Saúde Bucal na Clínica Odontológica. Editora artes Médicas.

Colli, A. S.; et al. (1975). Health survey of a group of adolescents in São Paulo, Brazil. Bol Oficina Sanit Panam. 79(5): 433-47.

Grego, A. J. (1968). Contribuição para o estudo da prevalência de anormalidades de oclusão em escolares do município de Araraquara, amarelos nascidos no Japão, amarelos nascidos no Brasil (Niseis) e brancos nascidos no Brasil. Suas relações com a higiene oral e as gengivites. RevFacFarmOdontol Araraquara. 2(1): 83-110.

Machado, C. V., Lima, L. D., \& Baptista, T. W. F. (2017). Políticas de saúde no Brasil em tempos contraditórios: caminhos e tropeços na construção de um sistema universal. Cad. Saúde Pública; 33 (2): S143-69.

Martins, A. M. E. B. L., et al. (2005) Levantamentos epidemiológicos brasileiros das condições de saúde bucal. Unimontes Científica; 7 (1).

Narvai, P. C., Frazão, P., \& Castellanos, R. A. (1999). Declínio na experiência de cárie em dentes permanentes de escolares brasileiros no final do século XX Odontologia e sociedade; $1(1 / 2): 25-29$.

Oliveira, A. G. R. C., et al. (1998). Levantamentos epidemiológicos em saúde bucal: análise da metodologia proposta pela Organização Mundial da Saúde. Rev. Bras. Epidemiol; 1(2).

Pereira, A. C., Frias, A. C., \& Vieira, V. (2016). Pesquisa estadual de saúde bucal: relatório final: Livro novo.

Pereira, A. C., \& Silva, R. P. (2009). Levantamentos epidemiológicos em odontologia. In: Pereira AC. Tratado de Saúde Coletiva em Odontologia. Napoleão, 704.

Pereira, M. G. (2000). Epidemiologia: teoria e prática. Ed. Guanabara Koogan.

Pinto, V. G. (2000). Saúde Bucal Coletiva. (4a ed.), Editora Santos.

Pinto, V. G. (1983). Saúde bucal no Brasil. Rev. Saúde Pública; 17: 316-27.

Pinto, V. G. (1996). Estudo epidemiológico sobre a prevalência da cárie dental em crianças de 3 a 14 anos, Brasil, 1993. SESI-DN.

Roncalli, A. G., Côrtes, M. I. S., \& Peres, K. G. (2012). Epidemiologia em saúde bucal e vigilância. Cad. Saúde Pública; 28: S58-S68.

Roncalli, A. G. (1998). Perfil Epidemiológico de Saúde Bucal no Brasil 1986-1996. Natal. http://www.angelonline.cjb.net

Rosendo, R. A., Sousa, J. N. L., Abrantes, J. G. S., Cavalcante, A. B. P., \& Ferreira A. K. T. F. (2017). Autopercepção de saúde bucal e seu impacto na qualidade de vida em idosos: uma revisão de literatura. RSC online; 6(1): 89-102.

Frias, A. C. Secretaria da Saúde de São Paulo. (2015). SB São Paulo 2015 - Pesquisa Estadual de Saúde Bucal - Relatório Final. 2015http://www.saude.sp.gov.br/ses/perfil/profissional-da-saude/areas-tecnicas-da-sessp/saude-bucal/sb-sao-paulo-2015-pesquisa-estadual-de-saude-bucalrelatorio-final.

São Paulo. Secretaria de Estado da Saúde. DIR-I - Capital. Faculdade de Saúde Pública da Universidade de São Paulo. (1999). Condições em saúde bucal: Estado de São Paulo, DIR-I, 1998: Relatório. São Paulo: DIR-I.

Secretaria da Saúde de São Paulo. (2015). SB São Paulo 2015 - Pesquisa Estadual de Saúde Bucal - Relatório Final. http://www.saude.sp.gov.br/ses/perfil/profissional-da-saude/areas-tecnicas-da-sessp/saude-bucal/sb-sao-paulo-2015-pesquisa-estadual-de-saude-bucalrelatorio-final.

World Health Organization. (1997). Oral healthsurveys: basicmethods. (4a ed.), ORH/EPID.

Zanetti, C. H. G. (1993). As marcas do mal-estar social no sistema Nacional de Saúde: o caso das políticas de saúde bucal, no Brasil dos anos 80 . Rio de Janeiro, 122p. Dissertação (Mestrado) - Escola Nacional de Saúde Pública, FIOCRUZ. 